\title{
Mathematical Programming for the Scheduling of Elective Patients in the Operating Room Department
}

\author{
Y.Y. Tan ${ }^{1}$, T. Y. ElMekkawy ${ }^{1}$, Q. Peng ${ }^{1}$, L. Oppenheimer ${ }^{2}$ \\ ${ }^{1}$ Department of Mechanical and Manufacturing Engineering, University of Manitoba, \\ 15 Gilson Street, Winnipeg, MB, R3T 5V6, Canada \\ ${ }^{2}$ Department of Surgery, Faculty of Medicine, University of Manitoba, \\ 727 McDermot Avenue, MB, R3E 3P5, Canada
}

\begin{abstract}
The Health Sciences Centre (HSC) in Winnipeg handles a large portion of the surgical patients in the province of Manitoba, along with Northwestern Ontario, and Nunavut. After an extensive analysis of the surgical patient flow at the facility, it became evident that the Operating Room (OR) scheduling system is one of the major sources of artificial variation in this flow, affecting both the pre and postoperative departments. A two-stage multi-objective mathematical model is proposed for the scheduling of elective cases. Using data obtained from all elective cases actually scheduled during a five week period at the HSC, five weekly OR schedules are generated using the first stage of the proposed mathematical model. The results of the model's schedules are compared to current schedules employed at the HSC, to verify its effectiveness and assess the improvements that can be achieved.
\end{abstract}

\section{Introduction}

In most healthcare facilities, the operating room (OR) department is a major bottleneck and represents a large portion of costs. The scheduling of cases in the OR is a balancing act between reducing costs and providing quality care in a timely, effective manner.

The master surgical schedule is a cyclic timetable that consists of blocks representing staffed OR hours. These blocks define the number of operating theatres available in an institution, the time that each theatre will be open, and the surgical groups or services (e.g. neurology, cardiology, etc.) that will be having their cases scheduled into each block. The cycle time for this schedule is usually one week, two weeks, or one month.

Once the master surgical schedule has been created, each surgical group chooses the cases they want scheduled into their blocks. Usually, each surgical group is given the responsibility for filling up their allocated block time. If a surgical group consists of more than one surgeon, cases may be scheduled on a first come first serve basis, or the surgeons in the group may have specific times allocated to them with which they can schedule cases as they please. This results in daily OR schedules, which list the actual operations to be performed, start and end times, operating theatres, etc.

There are a few papers that address the creation of master surgical schedules while considering bed occupancy. However, they make very simplistic assumptions because they can only estimate the types of cases that will be demanded in the future. The authors of [1] proposed and evaluated several mixed integer programming and simulated annealing models. They assumed that the schedule consisted of blocks allocated to surgeons, where each surgeon only performs one case type. In reality, most surgeons perform a variety of cases, each with different case durations and length of stays (LOS). The work of paper [2] also developed a mathematical linear programming model for creating weekly schedules that generate maximal revenue while taken the necessary constraints into account (e.g. OR time, bed numbers). The method assigns OR time to different services on different days of the week by considering each service's LOS. However, the LOS for patients in the same service can vary significantly, with some taking only one day while others may take two weeks. When surgeons are given control over choosing the operation dates for their cases, their choices constrain how the OR schedules will be created. Furthermore, the master surgical schedule may not result in the bed occupancy volumes anticipated because of the simplistic assumptions that were employed when it was developed. Because of this, the proposed mathematical model described in this paper was designed to generate weekly OR schedules with full 
control over operating theatre, date, and start time assignments for each case.

In literature, the majority of papers who create weekly OR schedules do not directly take post-operative bed occupancy volumes into account. In the papers presented in [3] and [4], patients are known to have arrived, or have been asked to arrive, at the hospital on a particular date. The task is to then schedule the patients so that the costs incurred by the patients waiting in the hospital for surgery are reduced. However, they do not take into account the time that the patient will be spending in the hospital after surgery occurs, and there are no calculations on whether or not enough beds will be available to accommodate all of those patients who are in the hospital or are scheduled to arrive.

The proposed mathematical model is based on the surgical patient flow at the HSC, Winnipeg, and was developed in conjunction with a research project that the HSC initiated. The main objective of the project was to analyze the facility's surgical patient flow and generate ideas on how to improve it. After several months of analysis, it was determined that one of the major issues that was impeding patient flow was the way that elective patients are scheduled. Indeed, these results were not surprising, considering that many papers in literature $[5,6]$ have cited that artificial variation, a major barrier to smooth patient flow, is commonly caused by wide variations in elective surgical admissions.

The model differs from all of the papers found in literature in that weekly schedules are generated with not only balanced daily patient volumes and utilized OR time in mind, but also the bed occupancy throughout the entire hospital, including the preoperative, recovery room (addressed in stage 2), and post-operative units. Furthermore, the majority of papers dealing with either weekly or daily OR schedules do not distinguish between different patient types and assume that all patient take the same path throughout the hospital. At the HSC, however, there are different types of patients who will take different paths, depending on their patient type. Besides the standard constraints that can be found in most papers with respect to resource availability (surgeon, operating theatre, equipment, etc.), there are additional constraints that have been included in the proposed model that are specific to HSC.

The rest of this paper is divided into six sections and because of space limitations, concentrates on the first stage of the mathematical model. Section 2 delivers the background information that is necessary to understand the basics of the different elective patient types at HSC, while Section 3 explains how they are scheduled in the OR department. Section 4 introduces the concept for the proposed multi-objective mathematical model and provides a detailed description of the first stage. In Section 5, the computational experiments and results from the first stage of the model are presented. Finally, concluding remarks, future work, and possible extensions of this model are given in Section 6.

\section{Surgical Patient Flow at the HSC}

The HSC in Winnipeg is the centre that handles the majority of all major elective and emergent surgical cases in the entire province of Manitoba, Northwestern Ontario, and Nunavut. Each elective surgical patient belongs to a particular service (e.g. neurosurgery, dental, orthopaedic), dependant on the type of surgery they require, and are sent to the unit corresponding to their service. If there are no beds available in a unit, patients are placed "off-service", meaning that they are sent to a unit corresponding to a different service. At the HSC, elective patients are classified as Inpatients, Same Day (SD) patients, Overnight patients, or Same Day Admission (SDA) patients. Patients admitted a day or more pre-operatively, as specified by their surgeon, can only be classified as Inpatients. The other three patient types are all admitted on the day of surgery and classified according to their expected discharge time. Patients expected to be discharged on the same day of surgery are classified as SD patients, Overnight patients are expected to be discharged by the next morning after surgery, and SDA patients are expected to be discharged one or more days after surgery.

Pre-operatively, Inpatients go to an inpatient unit corresponding to their service, Overnight patients go to B3, and SD and SDA patients go to MS3. Except for Inpatients, most patients are asked to arrive at their pre-operative units two hours before their surgical time. From their pre-operative units, all patients head to the OR department for surgery. From there, most patients go to the PACU for recovery. Afterwards, Inpatients, $\mathrm{SD}$ patients, and Overnight patients return to their pre-operative unit (which is now also their post-operative unit), while SDA patients go to the inpatient unit corresponding to their service. No other transfers usually take place before discharge occurs.

\section{Operating Room Scheduling at the HSC}

Currently at the HSC, there is one slate for elective patients and one slate for emergency patients during the week. On the weekends there is no elective slate and only emergency procedures are performed. In the first stage of the elective OR scheduling process at 
HSC, elective OR time is allocated to the different services and a master surgical schedule is drawn up. Each block in the master surgical schedule corresponds to a particular day in a particular operating theatre, and is allocated to a specific service. The managers of each service split up their blocks among the surgeons under their service. Surgeons then schedule cases into the times given to them in whatever manner they like. However, they do follow the rule that SD patients should be scheduled before their SDA patients. This is so that schedule interruptions due to lack of available post-operative beds for SDA patients will be reduced.

\section{Two-Stage Mathematical Model}

This paper presents a method for generating weekly elective operating room schedules in two stages, based upon the elective patient flow at HSC. The model works by considering all of the cases that need to be scheduled in the schedule week and assigns them to different days, operating theatres, and times so that a complete schedule is created. In the first stage, cases are assigned to the different days of the schedule, while taking into account case needs, such as pre and post-operative bed requirements, and constraints such as operating theatre, surgeon, and patient availability. In the second stage, each case is then assigned an operating theatre and an operating time. Because of space limitations, only the first stage will be detailed.

\subsection{Lexicographic Goal Programming}

The selection of a particular multi-objective method is driven by the type of information available and the specific characteristics of the problem. Taking into consideration the proposed mathematical model, goal programming (GP) was chosen to be the multiobjective optimization method. This is because GP can model the multiple objectives and aspects of the situation in a sufficiently realistic manner. Also, it is robust and efficient enough to be used in daily operation. GP is also used when deterministic optimization is acceptable given the relatively short time horizon for operation modeling, and this fits the problem at hand. Other multi-objective approaches could be used which could improve on one of these aspects at the expense of others, but GP strikes a balance appropriate for operation scheduling.

Lexicographic GP is used when no trade-off between criteria is allowed. Because the objectives of the proposed model have very obvious and different priority levels, lexicographic GP will be the method of choice. In this method, each problem addressed is a conventional, single-objective model. As such, the approach is appropriate for linear, integer and discrete, and nonlinear models. The primary advantage of this approach is that one may utilize any existing commercial single-objective software package as the primary problem-solving component. Therefore, the effort required to develop extremely efficient software in support of this approach is minimal. As a result, one may solve, with equivalent computational efficiency, problems of sizes equal to those solved by means of the supporting single-objective code.

The approach begins by addressing the portion of the model associated with priority level one as the single achievement term, which results in a conventional linear programming (LP) model. After solving, the approach proceeds to the next priority level and a new LP model is constructed with the terms in the second priority level turned into the single achievement function, while the previous solution for the first priority level is turned into a constraint. It is included to make sure that the solution previously derived is not degraded. In essence, the process is repeated until convergence is reached. Convergence is obtained when either the LP models for all priority levels have been examined or when the program is "fixed", where the value of structural variables become fixed at a certain stage, and will not change regardless of any remaining priority levels.

\subsection{Mathematical Model for Stage 1}

In the first stage of the mathematical model, surgeons with OR time in the scheduled week present the cases that they wish to schedule, provided that the total duration of all of their cases does not exceed their allocated OR time. The model then assigns the cases to the different days of the schedule week and determines the patient type that each case will be classified under. The solution obtained is based on seven goals, categorized into three priority levels. The first priority level is the most important, followed by the second, and finally the third. The goals, and their priority levels, were determined after an assessment of the factors affecting the patient flow at the HSC.

The first priority levels deals with the bed occupancy volumes throughout the post-operative units, where the three goals are to reduce the bed occupancy exceeded by each service on each day, balance the number of beds occupied by each service on each day, and minimize the bed occupancy on the days following the schedule week. The first goal decreases the number of patients that must be placed off-service due to a lack of beds, which often results in unnecessary transfers between different units throughout the hospital. The second goal achieves 
more constant daily bed occupancy volumes caused by the elective slate, and hence reduces artificial variability which often leads to delays, such as patients having to be put on hold because there are no postoperative beds available for them on busy days. The third goal reduces the impact of the scheduled week's elective slate on the days following the schedule, in terms of bed occupancy volumes. In this way, future schedules can be created with minimal disturbance from the previous weeks. This also forces bed utilization in the schedule week to be as high as possible, while still maintaining the other goals.

In the second priority level, the first goal reduces under-utilized OR time, which results in wasted resources, and overtime, which can lead to delays, increased costs, and staff dissatisfaction. The second goal minimizes the maximum number of days that any surgeon is scheduled to operate on, and ensures that no single surgeon is operating on an unnecessarily large number of days. This goal was added with surgeon satisfaction in mind.

The third priority level considers the daily patient volumes in the main pre-operative unit, MS3, and the OR department. The first goal balances the number of patients who will be arriving in MS3 pre-operatively, so that the unit experiences more constant daily patient volumes. This reduces the number of days with a high patient volume, which often results in delays that can affect the OR department. Similarly, the second goal achieves more constant daily patient volumes on the elective slate, which subsequently smooths the workload in all units. This goal is especially beneficial for the OR department and the PACU because during the day, the majority of patients received in both units is primarily made up of elective patients. When those units experience a high volume of patients, resources such as staffing, beds, and equipment may be inadequate, which usually causes delays.

It is assumed that patients follow the path that is currently taken by the majority of patients at the HSC, which is dependant upon their patient type. The solution obtained must satisfy a variety of constraints, some of which are unique to HSC. For example, elective cases can only be scheduled from Monday to Friday. MS3 has opening and closing times, while B3 needs to discharge their Overnight patients by the next morning in order to make room for new admissions.

Because of the way the model is designed, it replaces the need for the master surgical schedule. However, the amount of allocated OR time for each surgeon still needs to be determined, which is outside the scope of this model, and it is assumed that these values are known by the time the model is run.

\subsubsection{Notations}

The stage 1 mathematical model employs the following notations:

MS3 The closing time of the unit, in half hours

B3 The time at which new Overnight patients must be admitted, Monday to Friday

Inp $i \quad 1$ if case $i$ must be scheduled as an Inpatient; 0 otherwise

where $i=1, \ldots, \mathrm{n}$ (n $=$ number of cases to be scheduled)

Pre $_{i h} \quad 1$ if the patient of case $i$ requires to be admitted on day $h ; 0$ otherwise

where $h=1, \ldots, \mathrm{e}(\mathrm{e}=$ number of days before surgery)

$\operatorname{Dur}_{i} \quad$ The surgical duration of case $i$, in half hours

$\operatorname{Rec}_{i} \quad$ The recovery duration of case $i$, in half hours

Post $_{i}$ The post-operative duration of case $i$, in half hours, if less than 24 hours; 50 otherwise

Tot $_{i}$ The total duration of case $i$, including surgery, recovery, and post-operative care $\left(D u r_{i}+\operatorname{Rec}_{i}\right.$ + Post $_{i}$ )

$\mathrm{LOS}_{i}$ The post-operative length of stay for case $i$, in days, including the day of surgery

$\mathrm{OFF}_{i} 1$ if case $i$ cannot be placed off-service postoperatively; 0 otherwise

$\operatorname{Ser}_{i k} \quad 1$ if case $i$ is under service $k$; 0 otherwise

$\operatorname{Sur}_{i s} \quad 1$ if case $i$ is under surgeon $s ; 0$ otherwise where $s=1, \ldots, \mathrm{p}(\mathrm{p}=$ number of surgeons with cases)

$\mathrm{All}_{s} \quad$ The total OR time allocated to surgeon $s$ for the week's schedule

Over $_{j}$ The number of beds available for overnight patients in $\mathrm{B} 3$ on day $j$

where $j=1, \ldots, \mathrm{m}(\mathrm{m}=$ number of days in the schedule)

$\operatorname{Bed}_{g k}$ The number of beds available on day $g$ for cases under service $k$

where $g=1, \ldots, t(\mathrm{t}=$ number of days affected by the schedule)

where $k=1, \ldots, \mathrm{f}(\mathrm{f}=$ number of services $)$

$\psi_{g k} \quad$ The number of beds occupied on day $g$ by patients under service $k$, scheduled during the previous week

$\varphi_{g k} \quad$ The number of beds occupied on day $g$ by patients under service $k$ who cannot be placed off-service, scheduled during the previous week

$\mathrm{A}_{i g} \quad 1$ if case $i$ will occupy a bed on day $l ; 0$ otherwise

where $l=1, \ldots, \mathrm{q}(\mathrm{q}=$ number of days after surgery, including the day of surgery)

$\mathrm{B}_{j s} \quad 1$ if surgeon $s$ is available on day $j ; 0$ otherwise 
$\mathrm{C}_{i j} \quad 1$ if the patient of case $i$ is available on day $j ; 0$ otherwise

Open $_{j o}$ Time opening time on day $j$ for operating theatre $o$

where $o=1, \ldots, \mathrm{r} \quad(\mathrm{r}=$ number of staffed operating theatres)

Close $_{j o}$ The closing time on day $j$ for operating theatre

$o$

$\mathrm{D}_{j o} \quad$ Total OR time available on day $j$ for operating theatre $o$

$\operatorname{Max}_{j}$ The maximum OR time available by any operating theatre on day $j$

$\mathrm{Ava}_{j}$ The total OR time available on day $j$

$\mathrm{OR}_{i o} \quad 1$ if case $i$ can be performed in operating theatre $o$; 0 otherwise

$\mathrm{ORs}_{i}$ The number of operating theatres that case $i$ can be performed in

$\mathrm{Equ}_{j k}$ The number of equipment/instrumentation sets available on day $j$ for cases under service $k$

The solution to the stage 1 mathematical model is represented by the following variables:

$\mathrm{w}_{i j} \quad 1$ if case $i$ is assigned to day $j$; 0 otherwise

$\mathrm{x}_{i} \quad 1$ if case $i$ is scheduled as a SD patient; 0 otherwise

$\mathrm{y}_{i} \quad 1$ if case $i$ is scheduled as a Overnight patient; 0 otherwise

$\mathrm{z}_{i} \quad 1$ if case $i$ is scheduled as a SDA patient; 0 otherwise

$\mathrm{u}_{g k}$ The number of beds occupied on day $g$ by patients from service $k$, who cannot be placed off-service post-operatively

where $g=1, \ldots, t(t=$ number of days being considered)

$\mathrm{v}_{g k}$ The number of beds occupied on day $g$ by patients from service $k$

use $_{j}$ The total OR time used on day $j$

$\omega_{j s} \quad 1$ if surgeons $s$ is scheduled to operate on day $j ; 0$ otherwise

$\mathrm{ms}_{j}$ The number of cases scheduled on day $j$, whose patients will need to go to MS3 pre-operatively

num $_{j}$ The number of cases scheduled on day $j$

The following variables were used in order to transform the mathematical model into a linear program:

$\mathrm{xa}_{i j} \quad 1$ if case $i$ is assigned to day $j$ and scheduled as an SD patient; 0 otherwise

$\mathrm{ya}_{i j} \quad 1$ if case $i$ is assigned to day $j$ and scheduled as an Overnight patient; 0 otherwise

$\mathrm{za}_{i j} \quad 1$ if case $i$ is assigned to day $j$ and scheduled as an SDA patient; 0 otherwise

\subsubsection{Stage 1 Model}

Min $z=\left\{\left(10 b_{1 a}+5 b_{1 b}+b_{1 c}\right),\left(b_{2 a}+b_{2 b}\right),\left(b_{3 a}+b_{3 b}\right)\right\}$

subject to:

$$
\begin{aligned}
& w_{i j} S_{u r} \leq B_{j s} \\
& \forall i \in[1 . . n], \forall j \in[1 . . m], \forall s \in[1 . . p] \\
& w_{i j} \leq C_{i j} \quad \forall i \in[1 . . n], \forall j \in[1 . . m] \\
& D_{j o}=\text { Close }_{j o}-\text { Open }_{j o} \forall j \in[1 . . m], \forall o \in[1 . . r]
\end{aligned}
$$

$\operatorname{Max}_{j} \geq D_{j o} \quad \forall j \in[1 . . m], \forall o \in[1 . . r]$

$$
\sum_{i=1}^{n} w_{i j} \operatorname{Sur}_{i s} \text { Dur }_{i} \leq 1.2 \operatorname{Max}_{j}
$$$$
\forall j \in[1 . . m], \forall s \in[1 . . p]
$$

$$
\sum_{i=1}^{n} \operatorname{Sur}_{i s} \text { Dur }_{i} \leq A l l_{s} \quad \forall s \in[1 . . p]
$$

$$
\sum_{i=1}^{n} w_{i j} \operatorname{Ser}_{i k} \leq E q u_{j k} \quad \forall j \in[1 . . m], \forall k \in[1 . . f]
$$

$$
A v a_{j}=\sum_{o=1}^{p} D_{j o} \quad \forall j \in[1 . . m]
$$

$$
\sum_{i=1}^{n} D_{i} \leq \sum_{j=1}^{m} A v a_{j}
$$

$\sum_{\substack{i=1 \\ \text { ORs }}}^{n} w_{i j} \operatorname{Dur}_{i} O R_{i o} \leq D_{j o} \quad \forall j \in[1 . . m], \forall o \in[1 . . r]$

$$
\sum_{i=1}^{n} y a_{i j} \leq O V E R_{j} \quad \forall j \in[1 . . m]
$$

$$
\begin{aligned}
& \operatorname{Tot}_{i}=\text { Dur }_{i}+\operatorname{Re} c_{i}+\text { Post }_{i} \forall i \in[1 . . n] \\
& y a_{i j}=0 \\
& \quad \forall i \in[1 . . n] / \text { Inp }_{i}=0 / \text { Tot }_{i} \geq B 3, \\
& \forall j \in[1 . . m]
\end{aligned}
$$




$$
\begin{aligned}
& x a_{i j}+y a_{i j}=w_{i j} \\
& \forall i \in[1 . . n] / \operatorname{Inp}_{i}=0 / \operatorname{Tot}_{i}<M S 3, \\
& \forall j \in[1 . . m] \\
& y a_{i j}+z a_{i j}=w_{i j} \\
& \forall i \in[1 . . n] / \operatorname{Inp}_{i}=0 / \operatorname{Tot}_{i} \geq M S 3, \\
& \forall j \in[1 . . m] \\
& b_{1 a} \geq b_{1 a}^{-} \\
& b_{1 b}^{-}-b_{1 b}^{+} \geq v_{g k}-v_{a k} \\
& \forall g, a \in[1 . .7] / g>a, \forall k \in[1 . . f] \\
& b_{1 b} \geq b_{1 b}^{-}+b_{1 b}^{+} \\
& b_{1 c}=\sum_{g=8}^{t} \sum_{k=1}^{f} v_{g k} \\
& y a_{i j} \leq w_{i j} \\
& \forall i \in[1 . . n], \forall j \in[1 . . m](17) \\
& \forall i \in[1 . . n], \forall j \in[1 . . m] \quad(18) \\
& z a_{i j} \leq w_{i j} \\
& \forall i \in[1 . . n], \forall j \in[1 . . m] \text { (19) } \\
& x_{i}=\sum_{j=1}^{m} x a_{i j} \\
& \forall i \in[1 . . n] \\
& y_{i}=\sum_{j=1}^{m} y a_{i j} \\
& \forall i \in[1 . . n] \\
& z_{i}=\sum_{j=1}^{m} z a_{i j} \\
& \forall i \in[1 . . n] \\
& u_{g k}=\sum_{\substack{i=1 \\
O F F_{i}=1}}^{n} \sum_{j=1}^{m}\left(w_{i j}-x a_{i j}-y a_{i j}\right) \operatorname{Ser}_{i k}\left(\sum_{d=\text { dist. }(g, j)}^{w} \operatorname{LOS}_{i d}\right) \\
& +\sum_{\substack{i=1 \\
\text { Inp } \\
O F F_{i}=1}}^{n} \sum_{j=1}^{m} w_{i j} \operatorname{Ser}_{i k}\left(\sum_{e=\text { dist. }(g, j)}^{w} \operatorname{Pr} e_{i e}\right)+\psi_{g k} \\
& \forall g \in[1 . . t], \forall k \in[1 . . f] \\
& v_{g k}=\sum_{i=1}^{n} \sum_{j=1}^{m}\left(w_{i j}-x a_{i j}-y a_{i j}\right) \operatorname{Ser}_{i k}\left(\sum_{d=\text { dist. }(g, j)}^{w} \operatorname{LOS}_{i d}\right) \\
& +\sum_{\substack{i=1 \\
\operatorname{Inp}_{i}=1}}^{n} \sum_{j=1}^{m} w_{i j} \operatorname{Ser}_{i k}\left(\sum_{e=\operatorname{dist.} .(g, j)}^{w} \operatorname{Pr} e_{i e}\right)+\varphi_{g k} \\
& \forall g \in[1 . . t], \forall k \in[1 . . f] \\
& b_{1 a}^{-}-b_{1 a}^{+} \geq v_{g k}-B_{e d} \quad \forall g \in[1 . . t], \forall k \in[1 . . f]
\end{aligned}
$$




$$
\begin{aligned}
& w_{i j}, x_{i}, y_{i}, z_{i}, x a_{i j}, y a_{i j}, z a_{i j}, \omega_{j p} \in\{0,1\} \\
& \forall i \in[1 . . n], \forall j \in[1 . . m]
\end{aligned}
$$

In the objective function (1), $b_{1 a}, b_{1 b}$, and $b_{1 c}$ represent the three goals in priority level one. The first term, $b_{1 a}$, corresponds to the maximum number of beds exceeded by any service on any day. The second term, $b_{1 b}$, represents the maximum difference between the number of beds occupied by any service on any two days in the schedule week (Monday to Sunday). The third term, $b_{1 c}$, stands for the total number of beds occupied during the days after the schedule week. The two goals for priority level two are represented in the objective function (1) as $b_{2 a}$, and $b_{2 b}$. The term $b_{2 a}$ corresponds with the maximum deviation between the actual OR time (as dictated by the solution) and the available OR time on any day of the schedule, while the term $b_{2 b}$ represents the maximum number of days that any surgeon is scheduled to operate on. Finally, priority level three is represented by the terms $b_{3 a}, b_{3 b}$, and $b_{3 c}$ in the objective function (1). The term $b_{3 a}$ stands for the maximum difference between the amount of patients who will go to MS3 pre-operatively on any day of the schedule, while the term $b_{3 b}$ corresponds with the maximum difference between the total number of patients on any two days of the schedule.

Constraints (2) and (3) prohibit a case from being assigned to a day when the corresponding surgeon or patient is unavailable. Equation (4) calculates the amount of OR time that is available in each room on each day of the schedule, while equation (5) determines the maximum amount that can be found on each day of the schedule. Constraint (6) then specifies that the total duration of all cases belonging to a surgeon on a particular day cannot exceed $1.2 \%$ of the amount in equation (5). In this way, a surgeon will not end up having too many case hours assigned to a particular day which would result in overtime. Constraint (7) ensures that the required OR time for all of a surgeon's cases does not exceed the amount allocated to him/her in that schedule week. Constraint (8) allows cases to only be scheduled on days when the required equipment/instrumentation is available. Equation (9) calculates the total OR time needed for all cases that must be scheduled, and constraint (10) checks that this value does not exceed the total OR time available. For cases that can only be performed in the same, specific operating theatre, constraint (11) makes sure that if those cases are assigned to the same day, their total surgical duration does not exceed the total OR time available for that particular operating theatre. If this constraint did not exist, overtime for that particular operating theatre may be unavoidable in the second stage of the model. Constraint (12) specifies that on any day of the schedule, the total number of overnight patients cannot exceed the number of surgical beds available in B3. Constraints (13) through (22) determine whether a case, excluding the ones that must be scheduled as Inpatients, should be scheduled as an SD, Overnight, or SDA patient.

Equation (24) calculates the number of beds that will be occupied on each day by each service, while equation (23) calculates the same thing except only the patients who cannot be place-off service postoperatively are considered. In those equations, $d$ is the distance between day $g$ and day $j$, defined as $g-j+1$ if day $j$ precedes day $g, 0$ otherwise. Similarly, $e$ is the distance between day $g$ and day $j$, defined as $j-g$ if day $g$ precedes day $j, 0$ otherwise. Constraint (25) then ensures that for each day, there is an available bed for each patient that cannot be placed off-service.

Equations (26) to (27), (28) to (29), and (30) correspond to the first, second and third goal in priority level one, respectively. Equation (31) determines the total OR time that will be used on each day of the solution's schedule, which leads to the second goal of priority level two represented by equations (32) and (33). Equation (34) confirms that if a surgeon's case is assigned to a particular day, it means that the surgeon has to operate on that day. This is used in equations (35) to achieve the second goal in priority level two. Equation (36) determines the number of patients who will be going to MS3 preoperatively on each day of the schedule, and equation (39) similarly calculates the total number of patients on each day of the schedule. From there, equations (37) to (38), and (40) to (41), specify the first and second goals in priority level three, respectively. Equations (42) to (44) are the integrality constraints.

\section{Experimentation for Stage 1}

To evaluate the first stage of the proposed mathematical model, actual data from five weeks of elective surgical cases was collected from the HSC in order to create five different problems.

\subsection{Data}

The elective cases performed in each week represent the cases that need to be scheduled using the proposed model. For each case, their surgeon(s), service, and surgical duration was recorded. Estimated surgical 
durations were used in order to reflect how real scheduling problems do not have the luxury of knowing exact, actual durations beforehand.

Estimated recovery times in the PACU and postoperative units are not taken into consideration when scheduling at the HSC, so actual recovery durations were used in the model. Each patient's LOS was set to the nearest full day, up to a maximum of fourteen since most elective patients do not stay in the hospital for more than two weeks after surgery. All patients were modelled to have gone to the PACU after surgery, although patients who had surgery under local anaesthetic had their PACU recovery duration set to 0 . Patients admitted prior to the day of surgery were deemed as Inpatients, and their number of preoperative days was used in the model. Because elective inpatients are rarely asked to arrive more than a few days prior to surgery, the maximum number of pre-operative days assigned was set to four.

At the HSC during the period that data was collected for, surgical cases were classified into eleven different services. The OR department consisted of thirteen operating theatres, but two of them were utilized using the same OR team so the number of staffed operating theatres was set to twelve. The actual opening and closing times scheduled for each operating theatre on each day were used in the model, along with the closing time for MS3 and the time at which B3 had to admit new Overnight patients.

Because collecting data from five weeks of cases was such a large task, not all of the required information could be collected. Estimates were made on the days that each surgeon and patient were available for surgery. Also, the number of patients that could not be placed off-service was estimated by considering the services that patients belonged to. For example, most neurosurgery patients cannot be placed off-service after surgery, so they were all modelled as such. The elective surgical beds available for each service on each day were set at $80 \%$ of the amounts allocated by the hospital during that period. Reasonable estimates were made on the operating theatres could handle each case. For simplicity, it was assumed that each service has a specific set of equipment required for each of their cases, which cannot be shared with others.

\subsection{Results for Stage 1}

Table 1 presents the computational results for the five problems solved using stage 1 of the mathematical model. The term $n$ represents the number of cases to be scheduled in the schedule week, $p$ stands for the number of surgeons with cases, $Z$ corresponds to value of the objective found after the time given under $C P U$, and $B$ gives the best integer programming (IP) bound (i.e. the best solution that can theoretically be attained) that was reported by the solver. The solver was able to find the global optimum for all three priority levels in all five problems, except for priority level two in week 4 and priority level three in weeks 1 and 5. However, the solutions obtained for those weeks were very close to the best IP bounds and better than the results achieved by the schedule used at the HSC. Results are detailed in the following sections.

Table 1. Computational Results for Stage 1

\begin{tabular}{|c|c|c|c|c|c|c|}
\hline & n & $\mathbf{p}$ & $\begin{array}{c}\text { Priority } \\
\text { Level }\end{array}$ & $\begin{array}{c}\text { CPU } \\
\text { (min) }\end{array}$ & $\mathbf{Z}$ & B \\
\hline \multirow[t]{3}{*}{ Week1 } & \multirow[t]{3}{*}{126} & \multirow[t]{3}{*}{38} & 1 & 0.13 & 110 & 110 \\
\hline & & & 2 & 8.75 & 27 & 26.8 \\
\hline & & & 3 & 180 & $7 *$ & 1.5 \\
\hline \multirow{3}{*}{ Week2 } & \multirow[t]{3}{*}{148} & \multirow[t]{3}{*}{40} & 1 & 2.92 & 124 & 124 \\
\hline & & & 2 & 2.60 & 29 & 29 \\
\hline & & & 3 & 540 & 1 & 0.28 \\
\hline \multirow[t]{3}{*}{ Week3 } & \multirow[t]{3}{*}{101} & \multirow[t]{3}{*}{41} & 1 & 0.10 & 93 & 93 \\
\hline & & & 2 & 0.65 & 21 & 21 \\
\hline & & & 3 & 5.95 & 3 & 3 \\
\hline \multirow[t]{3}{*}{ Week4 } & \multirow[t]{3}{*}{135} & \multirow[t]{3}{*}{41} & 1 & 0.08 & 126 & 126 \\
\hline & & & 2 & 60 & $25^{*}$ & 24 \\
\hline & & & 3 & 1.45 & 0 & 0 \\
\hline \multirow[t]{3}{*}{ Week5 } & \multirow[t]{3}{*}{131} & \multirow[t]{3}{*}{39} & 1 & 0.10 & 89 & 89 \\
\hline & & & 2 & 5.88 & 28 & 27.4 \\
\hline & & & 3 & 60 & $4 *$ & 0 \\
\hline
\end{tabular}

*Best, but not necessarily optimal, solution found

\subsubsection{Priority Level One}

On any given day, patients are placed off-service when the number of beds available for their service is less than the number of patients of that service who are occupying beds. Table 2 shows how the mathematical model's solution for each problem resulted in a 53\% average reduction in the maximum number of patients that had to be placed off-service on any day during the schedule week, compared with the numbers achieved from the schedule that was actually used at the HSC. These results correspond with the first goal in priority level one, which should decrease delays associated with a high number of off-service patients, such as the number of unnecessary transfers of off-service patients between the different units throughout the hospital.

Table 2. Comparison between the Balance in OffService Bed Occupancy Volumes for Each Week

\begin{tabular}{|c|c|c|c|c|c|}
\hline \multirow{2}{*}{} & \multicolumn{5}{|c|}{$\begin{array}{c}\text { Maximum Number of Off-service Patients } \\
\text { from Any Service on Any Day }\end{array}$} \\
\cline { 2 - 6 } & Week1 & Week2 & Week3 & Week4 & Week5 \\
\hline HSC & 0 & 2 & 1 & 3 & 1 \\
\hline Model & 0 & 0 & 0 & 1 & 1 \\
\hline
\end{tabular}


Table 3 demonstrates how the mathematical model provided the same or better balanced bed occupancy for each week, than compared with the schedules actually used at the HSC. On average, the model was able to produce a $25 \%$ reduction in the maximum difference between the number of beds occupied by any service on any day. These results correspond with the second goal in priority level one and will reduce artificial variability caused by the elective OR slate, such as days when units reach full capacity and other days when those units are empty. This will improve the bed situation through the hospital.

Table 3. Comparison between the Balance in Bed Occupancy Volumes for Each Week

\begin{tabular}{|c|c|c|c|c|c|}
\hline \multirow{2}{*}{} & \multicolumn{6}{|c|}{$\begin{array}{c}\text { Maximum Difference between the Number of } \\
\text { Beds Occupied by Any Service on Any Two } \\
\text { Days }\end{array}$} \\
\cline { 2 - 6 } & Week1 & Week2 & Week3 & Week4 & Week5 \\
\hline HSC & 6 & 6 & 9 & 7 & 6 \\
\hline Model & 6 & 3 & 5 & 6 & 5 \\
\hline
\end{tabular}

Compared with the HSC schedule used, Table 4 depicts how the mathematical model generates a solution that produces a lower impact on the resultant bed occupancy during the days following the schedule. In fact, the model reduced the total bed occupancy on the days following the schedule week to an average of $18 \%$. These results correspond with the third goal in priority level one.

Table 4. Comparison between the Bed Occupancy Volumes following Each Schedule Week

\begin{tabular}{|c|c|c|c|c|c|}
\hline \multirow{2}{*}{} & \multicolumn{5}{|c|}{$\begin{array}{c}\text { Total Number of Beds Occupied during the } \\
\text { Days Following the Schedule Week }\end{array}$} \\
\cline { 2 - 6 } & Week1 & Week2 & Week3 & Week4 & Week5 \\
\hline HSC & 98 & 134 & 80 & 97 & 76 \\
\hline Model & 80 & 109 & 68 & 86 & 54 \\
\hline
\end{tabular}

Together, the first, second, and third goals in priority level one will create a schedule where bed utilization is high during the scheduled week, while maintaining a balanced occupancy in each unit and ensuring that the number of patients placed off-service remains as little as possible. In this way, the bed resources at the HSC will be used effectively.

\subsubsection{Priority Level Two}

Under-utilized OR time is defined as the positive deviation of available OR time minus utilized OR time, while overtime is defined as the negative deviation. At the HSC, cases are scheduled into the available OR time until there is none left, so theoretically there should not be any under-utilized OR time or overtime unless surgical duration estimates are wrong. The mathematical model, however, may generate a schedule with known under-utilized OR time or overtime beforehand since the model considers far more goals than just matching available and actual OR time. Table 5 demonstrates that for each week, the model was able to balance the overtime and underutilized OR time between each day to a maximum of two and a half hours during week 4, which was achieved using the first goal in priority level two.

Table 5. The Balance in Overtime and Underutilized OR Time for Each Week

\begin{tabular}{|c|c|c|c|c|c|}
\hline & Wee1 & Week2 & Week3 & Week4 & Week5 \\
\hline Overtime & 0 & 0 & 0 & 0 & 0 \\
\hline $\begin{array}{c}\text { Under- } \\
\text { utilized } \\
\text { Time }\end{array}$ & 1 & 3 & 1 & 5 & 2 \\
\hline
\end{tabular}

At the HSC, surgeons are given their assigned operation days and times a number of weeks beforehand so that they are able to schedule their other activities around those times. With this mathematical model, however, surgeons have to provide information about when they will be available to operate so that the model can fit their cases onto those days. Table 6 portrays how the model performs when trying to minimize the maximum number of days that any surgeon has to operate, which corresponds with goal two in priority level two.

Table 6. The Maximum Operation Days for Any Surgeon in Each Week

\begin{tabular}{|c|c|c|c|c|}
\hline \multicolumn{5}{|c|}{ Maximum Number of Operation Days for Any Surgeon } \\
\hline Week1 & Week2 & Week3 & Week4 & Week5 \\
\hline 3 & 5 & 4 & 4 & 3 \\
\hline
\end{tabular}

\subsubsection{Priority Level Three}

The final comparison between the schedule used at the HSC and the schedule obtained by the mathematical model is the difference between the total number of cases, and the number of patients who have to go to MS3 pre-operatively, on each day during the schedule week. Tables 7 and 8 illustrate how the numbers are more balanced when using the mathematical model than compared to the HSC schedule. The model reduced the variability between MS3 and total patient volumes by an average of $92 \%$ and $68 \%$, respectively This was accomplished using priority level three, and will result in a more balanced daily workload for all departments involved in the surgical patient flow, 
especially for MS3, the OR department, PACU. This will reduce artificial variability caused by varying patient volumes and will therefore reduce delays.

Table 7. Comparison between the Balance in MS3 Patient Volumes for Each Week

\begin{tabular}{|c|c|c|c|c|c|}
\hline \multirow{2}{*}{} & \multicolumn{5}{|c|}{$\begin{array}{c}\text { Maximum Difference between the Number of } \\
\text { MS3 Patients on Any Two Days }\end{array}$} \\
\cline { 2 - 6 } & Week1 & Week2 & Week3 & Week4 & Week5 \\
\hline HSC & 15 & 12 & 4 & 12 & 7 \\
\hline Model & 2 & 0 & 0 & 0 & 2 \\
\hline
\end{tabular}

Table 8. Comparison between the Balance in Total Patient Volumes for Each Week

\begin{tabular}{|c|c|c|c|c|c|}
\hline \multirow{2}{*}{} & \multicolumn{5}{|c|}{$\begin{array}{c}\text { Maximum Difference between the Total } \\
\text { Number of Patients on Any Two Days }\end{array}$} \\
\cline { 2 - 6 } & Week1 & Week2 & Week3 & Week4 & Week5 \\
\hline HCS & 11 & 15 & 4 & 12 & 6 \\
\hline Model & 5 & 1 & 3 & 0 & 2 \\
\hline
\end{tabular}

\section{Conclusion}

This paper described the first stage of a two-stage mathematical model for the scheduling of elective cases, based upon the elective surgical patient flow at the HSC in Winnipeg, Manitoba. In the first stage of the model, cases are assigned to the different days of the schedule week, while taking into account case needs and respecting constraints. The generated solution achieves goals that reduce artificial variability caused by varying bed occupancy and patient volumes due to the elective slate, in addition to minimizing overtime and under-utilized OR time.

The first stage of the mathematical model was tested using data from five consecutive weeks of elective cases at the HSC. The results obtained were then compared to the actual bed occupancy and patient volumes resulting from the schedules used at the HSC. Judging by the performance of the first stage of the mathematical model, it can be concluded that it was able to successfully achieve the intended goals.

There is the strong indication that the mathematical model will result in less artificial variability than compared to the way the facility currently schedules their elective cases, and will likely attain smoother surgical patient flow. With these promising results, experimentation for the second stage of the model can now be carried out. Even on its own, the first stage will be able to provide a good guideline to the HSC regarding which days certain cases should be performed in order to smooth bed occupancy and patient volumes while minimizing overtime and under-utilized OR time. The HSC can then carry out daily scheduling in the manner that they desire.

The proposed model was created specifically with the HSC in mind. However, it can be easily tailored to meet the needs of other facilities by adjusting or adding more constraints, fine-tuning or adding goals, or changing the order of the priority levels according to the modeller's preferences.

\section{Acknowledgements}

This research project was funded by the Winnipeg Regional Health Authority and Health Science Centre. The authors gratefully thank Helga Bryant and Leanne Bernaerdt for their cooperation and support.

\section{References}

[1] J. Belien, and E. Demeulemeester, "Building cyclic master surgery schedules with leveled resulting bed occupancy,", J.of AORN Journal, vol. 81, pp. 69-81, March 2005 .

[2] M.V. Calichman, "Creating an optimal operating room schedule,", Journal J. of Medical Systems, , vol. 27, pp. 580588, June 2003.

[3] A. Guinet, and S. Chaabane, "Operating theatre planning", International I. Journal J. of Production Prod. EconomicsEcon., vol. .85, pp. .69-81, 2003.

[4] S.N. Ogulata, and R. Erol, "A Hierarchical multiple criteria mathematical programming approach for scheduling general surgery operations in large hospitals,", Journal J. of Medical Systems, vol. .27, pp. .259-270, June 2003.

[5] Optimizing patient flow: moving patients smoothly through acute care settings. IHI Innovation Series white paper. Institute for Healthcare Improvement (IHI), Boston. [Online] Available: www.IHI.org 2003.

[6] C. Haraden, and R. Resar, "Patient flow in hospitals: understanding and controlling it better," Frontiers of Health Services Management, vol. 20, pp. 3-15, Summer 2004. 